\title{
Flux Pinning in a Superconductor by an Array of Submicrometer Magnetic Dots
}

\author{
J. I. Martín,* M. Vélez,* J. Nogués, ${ }^{\dagger}$ and Ivan K. Schuller \\ Physics Department, University of California-San Diego, La Jolla, California 92093-0319
}

(Received 10 March 1997)

\begin{abstract}
Triangular arrays of submicrometer magnetic dots, with typical spacing of 400-600 nm and diameters close to $200 \mathrm{~nm}$, have been fabricated by electron beam lithography to study pinning effects on $\mathrm{Nb}$ thin films. The resistivity versus magnetic field curves exhibit regular structure. Minima appear at constant field intervals, given by the lattice parameter of the dot array. The angular, current, and temperature dependencies of the resistivity imply synchronized pinning by the magnetic array which is relevant at high vortex velocities, when the order in the vortex lattice increases. [S0031-9007(97)03979-3]
\end{abstract}

PACS numbers: 74.60.Ge, 85.40.Ux

Flux pinning has been the subject of much interest both in high and low temperature superconductors because of their relevance for applications as well as from the fundamental point of view [1,2]. A rich variety of phenomena in vortex dynamics can be found depending on the type, strength, and correlation of the defects present in the superconducting material. A very useful tool to understand the interaction between vortices and material imperfections, and in this way enhance the critical current, consists in the introduction of controlled artificial pinning centers in the superconductor. Many types of random imperfections such as secondary-phase precipitates [3], cold work induced dislocations and strain [4], several kinds of radiation induced defects like heavy-ion columnar tracks [5], etc. can be used to address this problem.

A different approach is the fabrication of ordered defect arrays of well defined size and geometry, as, for example, spatially modulated superconducting alloys [6], thickness modulation [7], holes in superconducting networks [8], arrays of holes [9], or magnetic particles [10]. In this way, a whole range of new phenomena may appear associated with the matching of the vortex lattice and the artificial structure. However, only recently submicrometer lithographic techniques have been developed that allow reducing the size of pinning centers to a scale comparable to coherence lengths of conventional superconductors. Some possibilities that have been explored include regular lattices of holes on low $T_{C}$ superconducting films [11] or spatially modulated $e$-beam irradiation damage in $\mathrm{YBa}_{2} \mathrm{Cu}_{3} \mathrm{O}_{7}$ [12]. In all these cases normal regions are created throughout the material.

In this Letter, for the first time the pinning interaction between the vortex lattice and ordered arrays of submicrometer magnetic dots has been studied. Therefore, the superconducting thin film is continuous and pinning arises from the interaction with a periodic array of pinning centers not from the multiply connected geometry of the system. In this case, in a narrow temperature range, an evenly spaced regular array of minima appears in the resistivity versus perpendicular magnetic field. The periodicity of the minima is given by the field corresponding to the spacing of the array of magnetic dots. This indi- cates a collective locking of the flux lattice to the magnetic dot array. The detailed vortex arrangement has not been uniquely identified. Comparison with available theoretical models indicates a synchronized pinning by the magnetic dot array at high vortex velocities.

Magnetic dots approximately $200 \mathrm{~nm}$ in diameter and $40 \mathrm{~nm}$ in thickness were prepared on oxidized $\mathrm{Si}(100)$ substrates using the electron beam lithography. The desired pattern was defined on a PMMA resist layer; then the magnetic (Fe or Ni) layer was sputtered on top. This was followed by a lift-off process to obtain the triangular array $(50 \mu \mathrm{m} \times 50 \mu \mathrm{m})$ of magnetic dots. The lattice constant (d) of the triangular array was varied in the range 400$600 \mathrm{~nm}$. Figure 1 shows a scanning electron microscopy (SEM) image of a triangular Fe array with $d=600 \mathrm{~nm}$. After this, a $100 \mathrm{~nm}$ thick $\mathrm{Nb}$ film was sputtered on top. Optical lithography and reactive ion etching were used to define a $40 \mu \mathrm{m}$ wide bridge with a distance between the voltage contacts of $50 \mu \mathrm{m}$ for transport measurements [see Fig. 1(b)]. Note that in this geometry the Nb film is continuous so the pinning dots are not embedded within the $\mathrm{Nb}$, but are outside.

The dc measurements were performed in a helium cryostat with an $80 \mathrm{kG}$ superconducting magnet and a rotatable sample holder with a precision of $1^{\circ}$. The magnetic field is always applied perpendicular to the transport current, while the angle $\theta$ between the field and the film normal was varied to study the angular dependence. The lithographed $\mathrm{Nb}$ film on top of the magnetic array of dots exhibits metallic behavior from room temperature to the onset of superconductivity at $T=8.3 \mathrm{~K}$.

Figure 2 shows $\rho(B)$ for a sample with an array of $\mathrm{Ni}$ $\operatorname{dots}\left(d=410 \mathrm{~nm}, J=2.5 \times 10^{4} \mathrm{~A} / \mathrm{cm}^{2}\right)$ at $T=8.2 \mathrm{~K}$. In the mixed state $(8.1<T<8.3 \mathrm{~K})$, the resistivity versus magnetic field shows a set of minima regularly spaced at values $\left(B_{n}\right)$ of field. The intervals between two consecutive minima $\left(\Delta B_{0}\right)$ are constant, as shown in the inset of Fig. 2, with $\Delta B_{0}=141 \pm 4 \mathrm{G}$. This value may be related with the lattice parameter $d$ of the triangular magnetic array through the well known relation $a_{0}=1.075$ $\left(\Phi_{0} / B\right)^{1 / 2}$ [13], between the magnetic induction $(B)$, and the corresponding triangular vortex lattice constant $\left(a_{0}\right)$ 

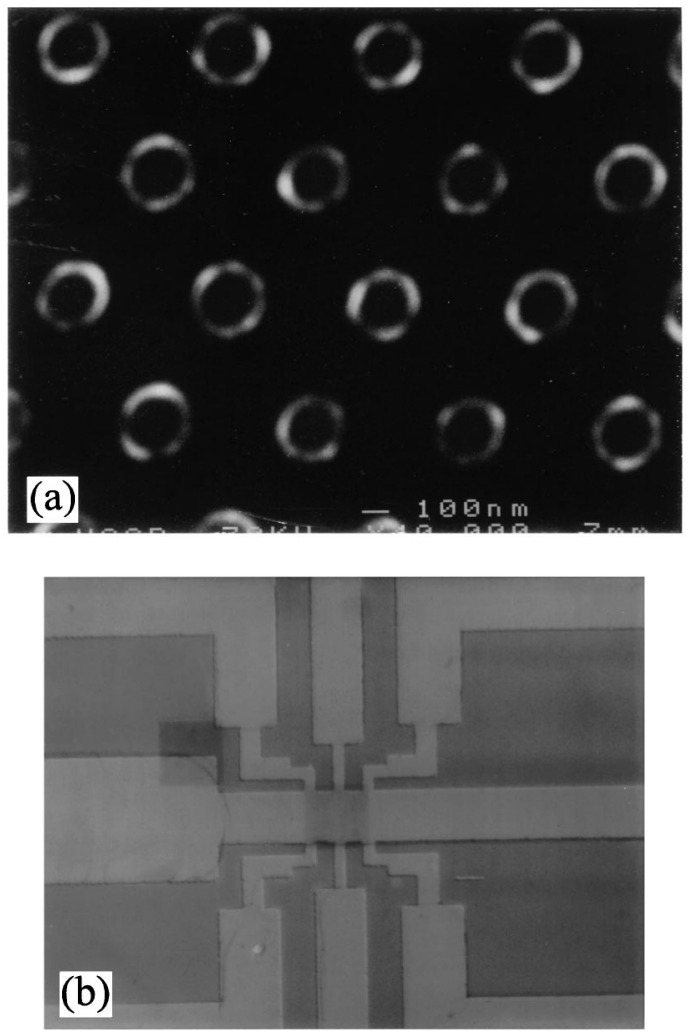

FIG. 1. (a) SEM image of a triangular array of Fe magnetic dots fabricated by $e$-beam lithography on a Si substrate. The lattice constant is $d=600 \mathrm{~nm}$ and the $\mathrm{Fe}$ dots have a radius $r=100 \mathrm{~nm}$. (b) Micrograph of the $\mathrm{Nb}$ bridge defined by optical lithography in the array region. The width is $40 \mu \mathrm{m}$ and the distance between the voltage contacts used for transport measurements is $50 \mu \mathrm{m}$.

with $\Phi_{0}$ the quantum of flux. Thus, the interval $\Delta B_{0}$ corresponds to $a_{0}=413 \pm 6 \mathrm{~nm}$, in good agreement with the spacing of the array $d=410 \pm 10 \mathrm{~nm}$. This implies that a minimum appears when there is an integer number of vortices per unit cell of the array of magnetic dots. We find that this structure in the dissipation is independent of the magnetic history of the dot array, i.e., thermally demagnetized or exposed to a magnetic field up to $10 \mathrm{kG}$, parallel or perpendicular to the sample plane. This lack of hysteresis in the interaction of the magnetic array and the superconducting film indicates that the stray field from the Ni dots does not play a crucial role. Therefore, the pinning interaction between the vortices and the magnetic dots is principally determined by the flux concentration in or around the magnetic material and/or the spatial modulation of the order parameter due to the ferromagnetic proximity effect, in a similar way to the effect of ferromagnetic pinning centers in bulk superconductors [14]. No indication of periodic pinning has been found in arrays of nonmagnetic dots measured at similar temperatures, current, and field ranges, suggesting that pinning due to the ordinary proximity effect is too weak to be observed. This confirms that the pinning produced by the array of $\mathrm{Ni}$ dots is mainly of magnetic origin.

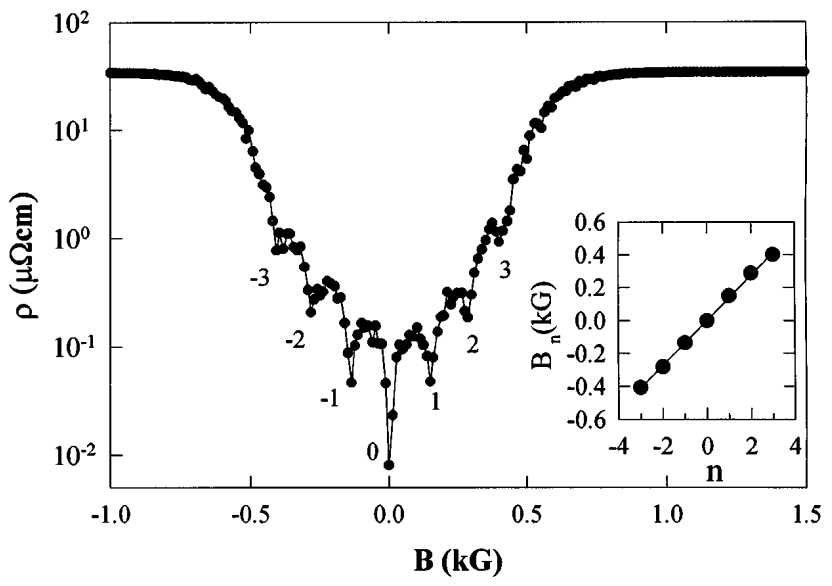

FIG. 2. Field dependence of the resistivity of a $\mathrm{Nb}$ thin film with a triangular array of $\mathrm{Ni}$ dots $(d=410 \mathrm{~nm})$, measured at $T=8.2 \mathrm{~K}$ and with $J=2.5 \times 10^{4} \mathrm{~A} / \mathrm{cm}^{2}$. Inset shows the position of the minima $B_{n}$ versus the index number $n$. The solid line is a fit to the expression $B_{n}=n \Delta B_{0}$ with $\Delta B_{0}=141 \pm 4 \mathrm{G}$.

Figure 3 shows the field dependence of the resistivity of the same $\mathrm{Nb}$ film as in Fig. 2, for different angles $\theta$ between the sample normal and the field direction. Sharp minima are observed in the whole angular range but the field intervals increase as the magnetic field is tilted away from the perpendicular direction. The critical field $H_{c 2}$ shows an angular dependence that is in good agreement with Tinkham's model for a superconducting thin film [13]. The angular dependence of the distance between minima is well described by $\Delta B_{0}(\theta)=\Delta B_{0}(0) / \cos (\theta)$ with $\Delta B_{0}(0)=138 \pm 3 \mathrm{G}$ (Fig. 3 inset). Note that the

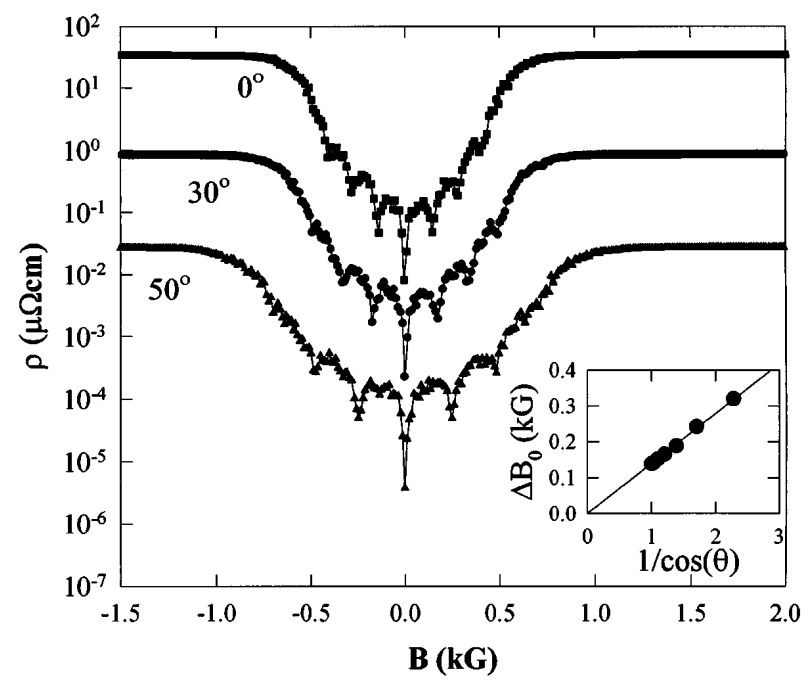

FIG. 3. Resistivity versus magnetic field curves for different angles $\theta$ between the field and the sample normal of a $\mathrm{Nb}$ thin film with a triangular array of $\mathrm{Ni} \operatorname{dots}(d=410 \mathrm{~nm})$, measured at $T=8.2 \mathrm{~K}$ with $J=2.5 \times 10^{4} \mathrm{~A} / \mathrm{cm}^{2}$. The curves have been vertically displaced for clarity. Inset shows the angular dependence of the distance between consecutive minima. The solid line is a fit to the expression $\Delta B_{0}(\theta)=\Delta B_{0}(0) / \cos (\theta)$ with $\Delta B_{0}(0)=138 \pm 3 \mathrm{G}$. 
two independent measurements of $\Delta B_{0}$ agree within the experimental error. This implies that the relevant quantity is the perpendicular magnetic field component $\left(B_{\perp}\right)$, so that only the number of vortices per unit cell of the periodic array is important.

The reduction in dissipation, at well defined values of the magnetic field, is present only for a limited range of currents and temperatures. Figure 4(a) shows $\rho(B)$ taken at different current densities in the range $J=$ $10^{3}-10^{5} \mathrm{~A} / \mathrm{cm}^{2}$. At the highest current (curve $A$ ) the resistivity is similar to that of the normal state and weakly field dependent. As the current density is reduced a periodic structure appears: first, two small features $(n=1)$ around zero field (curve $B$ ), then (curves $C$ and $D$ ); the minima become deeper and the matching effect can be observed for higher orders, up to $n=4$, for $J$ values
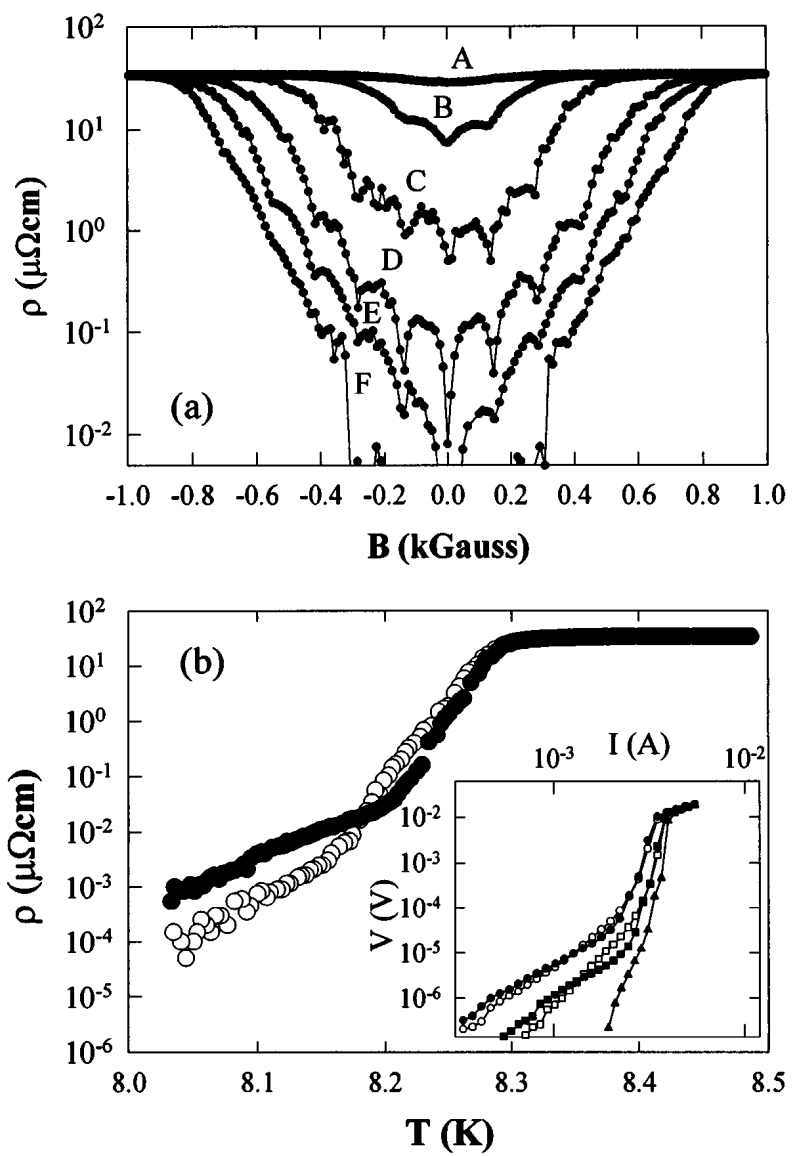

FIG. 4. (a) Field dependence of the resistivity of a $\mathrm{Nb}$ film with a triangular lattice of $\mathrm{Ni}$ magnetic dots $(d=$ $410 \mathrm{~nm}$ ) at $T=8.2 \mathrm{~K}$ for different values of the transport current: Curve $A, J=7.5 \times 10^{4} \mathrm{~A} / \mathrm{cm}^{2}$; curve $B, J=5.6 \times$ $10^{4} \mathrm{~A} / \mathrm{cm}^{2} ;$ curve $C, J=5 \times 10^{4} \mathrm{~A} / \mathrm{cm}^{2}$; curve $D, J=$ $2.5 \times 10^{4} \mathrm{~A} / \mathrm{cm}^{2}$; curve $E, J=1.25 \times 10^{4} \mathrm{~A} / \mathrm{cm}^{2}$; curve $F$, $J=2.5 \times 10^{3} \mathrm{~A} / \mathrm{cm}^{2}$. (b) Resistive transition in a logarithmic scale of the same sample as in (a), measured with $J=2.5 \times 10^{4} \mathrm{~A} / \mathrm{cm}^{2}$ and an applied perpendicular magnetic field of $70 \mathrm{G}$ (open symbols) and $140 \mathrm{G}$ (filled symbols). Inset shows the $I-V$ curves at $T=8.1 \mathrm{~K}$ measured for different fields: triangle, $0 \mathrm{G}$; open square, $110 \mathrm{G}$; filled square, $140 \mathrm{G}=B_{1}$; open circle, $250 \mathrm{G}$; filled circle, $280 \mathrm{G}=B_{2}$. around $2.5 \times 10^{4} \mathrm{~A} / \mathrm{cm}^{2}$. However, when the current density is further decreased (curve $E$ ) the periodic structure in the dissipation begins to be smeared out. Finally, for the lowest currents (curve $F$ ), the typical uniform superconducting film behavior is found, with a sharp onset of the resistivity for a magnetic field close to a matching value, but without any well defined features at the other matching positions. This reduction in the dissipation can also be observed in the $I-V$ characteristics [see Fig. 4(b) inset]. The $I-V$ curve measured at each matching field $B_{n}$ (filled symbols) crosses with the one obtained at lower field (hollow symbols) for high enough currents. These crossovers indicate a change in the dominant pinning mechanism as a function of the current.

We have observed that the current range where pinning by the magnetic array is more effective is also temperature dependent, and it is displaced to higher values as the temperature is reduced. This is shown [Fig. 4(b)] on a logarithmic plot of the resistivity, measured with $J=2.5 \times 10^{4} \mathrm{~A} / \mathrm{cm}^{2}$, in the presence of a constant magnetic field, for two different fields. The open and filled symbols correspond to $B=70$ and $140 \mathrm{G}$, respectively, that is, $B_{1} / 2$ and $B_{1}$, where $B_{1}$ is the first matching field. At low temperatures, the ordinary monotonic behavior is found and the resistivity increases with the magnetic field; however, at temperatures very close to the transition a distinct crossover is observed and $\rho\left(B_{1}\right)$ becomes smaller than $\rho\left(B_{1} / 2\right)$, corresponding to the minima in the dissipation shown in Fig. 4(a). In a similar way, Gurevich et al. [15] have recently reported resistivity oscillations as a function of a parallel magnetic field in a limited current range in $\mathrm{Nb}-\mathrm{Ti} / \mathrm{Cu}$ multilayers. However, on these multilayers the oscillation period is dependent on the total sample thickness, is current dependent, and disappears at high temperatures.

The experimental data clearly show an enhanced pinning by the magnetic dots and a definite relationship between the vortex lattice and the magnetic dot array. There are, however, two major issues which need further research and must be addressed: (a) the pinning mechanism and (b) the details of the vortex lattice arrangement.

The magnetic moment of an individual dot lies parallel to the substrate [16] due to their aspect ratio $c / t \approx 5$ (where $c=200 \mathrm{~nm}$ is the diameter and $t=40 \mathrm{~nm}$ is the thickness). Therefore, as the perpendicular field component is the relevant one, the pinning mechanism must be related to the higher magnetic permeability in the region of the dot. The perturbation created in a superconductor in the neighborhood of an embedded magnetic particle of radius comparable to the coherence length and magnetic moment $m$ has recently been calculated [17]. Depending on the value of $m$, it may be energetically favorable for the superconductor to create a vortex state with multiple quanta of flux around the magnetic particle. However, the $r / \xi$ ratio and the current dependence seem to rule out trapping of multiple vortices in each dot. Near $T_{c}, \xi$ becomes similar to the dot radius $r=100 \mathrm{~nm}[18] ; \xi=60 \mathrm{~nm}$ at 
$T=8.2 \mathrm{~K}$. Therefore, the maximum number of vortices pinned inside each dot is estimated as $r / 2 \xi \approx 1$, using as a first approximation the formula for a hole of similar size $[19,20]$.

The periodic resistivity dips of the $\mathrm{Nb}$ film with the lattice of magnetic dots (Figs. 2 and 3 ) are due to commensurability effects between the ordered magnetic dot array and the vortex lattice. Dips in the resistivity imply an enhanced pinning for particular values of the magnetic field. In the case of a triangular array of pinning centers, commensurability occurs with a regular triangular vortex lattice if $a_{0}=d, a_{0}=d / \sqrt{3}, a_{0}=d / 2, \ldots$ with matching fields at $B_{n}=n \Delta B_{0}$, with $n=1,3,4, \ldots$ Thus, no commensurability is expected for $n=2$ for a regular triangular lattice, in contrast with experimental observations. Recent numerical simulations [21] have shown that a triangular lattice of defects enhances the pinning force at $n=1,3,4$ for weak pinning centers. However, strong pinning sites may induce some pinning enhancement at $n=2$ [21]. An increased pinning may also occur at this second matching field if long range interactions are present in the vortex lattice, thus forming a lattice with one vortex per dot and one every other interstitial position in the center of the triangles, as observed by Lorentz microscopy [22].

The presence of enhanced resonant pinning, only at intermediate currents, indicates that at low vortex velocities, random defect pinning occurs, while at higher velocities, dynamical effects may become important. The moving lattice averages over the random pinning potential and vortexvortex interactions, producing the order in the lattice, may become dominant [23]. In fact, neutron diffraction experiments [24] have shown an increased order of the moving vortex lattice at high velocities. On the other hand, the interaction between the periodic array of dots and the vortex lattice could become more important at high velocities because periodic pinning cannot be averaged out, like random pinning. This enhancement of the interaction with the magnetic array, as the vortex lattice becomes more ordered, would give rise to the observed matching effect at intermediate currents.

In summary, we have studied the pinning effect of a triangular array of submicron magnetic dots fabricated by $e$-beam lithography on a superconducting $\mathrm{Nb}$ film. Sharp, regularly spaced minima found in the $\rho\left(B_{\perp}\right)$ curves are related to the pinning caused by the regular array of magnetic dots. This pinning is relevant in the temperature range where the coherence length $\xi$ is similar to the dot radius. The current dependence indicates that this behavior is produced by the synchronized pinning of the magnetic dot array at high vortex velocities when the order in the vortex lattice is enhanced.

This work has been supported by the U.S. Department of Energy, Air Force Office of Scientific Research, and the Spanish Ministerio de Educación y Cultura. We acknowledge useful discussions with D. Arovas, A. A. Abrikosov, R.C. Dynes, F. Hellman, V. Kogan, J. L.
Vicent, Y. Jaccard, and A. Hoffmann. We specially thank F. Sharifi for help with the electron beam lithography.

*On leave from Universidad Complutense, 28040 Madrid, Spain.

${ }^{\dagger}$ On leave from Universitat Autònoma de Barcelona, 08193 Barcelona, Spain.

[1] A. M. Campbell and J.E. Evetts, Adv. Phys. 21, 199 (1972).

[2] G. Blatter, M. V. Feigel'man, V.B. Geshkenbein, A. I. Larkin, and V.M. Vinokur, Rev. Mod. Phys. 66, 1125 (1994).

[3] C. Meingast and D.C. Larbalestier, J. Appl. Phys. 66, 5962 (1989).

[4] J. W. Ekin, J. Appl. Phys. 62, 4829 (1987).

[5] L. Civale, A. D. Marwick, T. K. Worthington, M. A. Kirk, J. R. Thompson, L. Krusin-Elbaum, Y. Sun, J. R. Clem, and F. Holtzberg, Phys. Rev. Lett. 67, 648 (1991).

[6] H. Raffy, J. C. Renard, and E. Guyon, Solid State Commun. 11, 1679 (1972).

[7] O. Daldini, P. Martinoli, J. L. Olsen, and G. Berner, Phys. Rev. Lett. 32, 218 (1974).

[8] B. Pannetier, J. Chaussy, R. Rammal, and J. C. Villegier, Phys. Rev. Lett. 53, 1845 (1984).

[9] A. T. Fiory, A. F. Hebard, and S. Somekh, Appl. Phys. Lett. 32, 73 (1978).

[10] Y. Otani, B. Pannetier, J.P. Nozières, and D. Givord, J. Magn. Magn. Mater. 126, 622 (1993).

[11] M. Baert, V. V. Metlushko, R. Jonckheere, V. V. Moshchalkov, and Y. Bruynseraede, Phys. Rev. Lett. 74, 3269 (1995).

[12] J.-Y. Lin, M. Gurvitch, S. K. Tolpygo, A. Bourdillon, S. Y. Hou, and J. M. Phillips, Phys. Rev. B 54, R12717 (1996).

[13] M. Tinkham, Introduction to Superconductivity (McGrawHill, New York, 1975), p. 134.

[14] N. D. Rizzo, J. Q. Wang, D. E. Prober, L. R. Motowidlo, and B. A. Zeitlin, Appl. Phys. Lett. 69, 2285 (1996).

[15] A. Gurevich, E. Kadyrov, and D. C. Larbalestier, Phys. Rev. Lett. 77, 4078 (1996).

[16] B. D. Cullity, Introduction to Magnetic Materials (Addison-Wesley, Reading, MA, 1972), Chap. 9.

[17] I. K. Marmorkos, A. Matulis, and F. M. Peeters, Phys. Rev. B 53, 2677 (1996).

[18] I. Banerjee, Q.S. Yang, C. M. Falco, and I. K. Schuller, Phys. Rev. B 28, 5037 (1983).

[19] G. S. Mkrtchyan and V. V. Shmidt, Sov. Phys. JETP 34, 195 (1972).

[20] V. V. Moshchalkov, M. Baert, V. V. Metlushko, E. Rossel, M. J. van Bael, K. Temst, R. Jonckheere, and Y. Bruynseraede, Phys. Rev. B 54, 7385 (1996).

[21] C. Reichhardt, J. Groth, C. J. Olson, and F. Nori, Phys. Rev. B 54, 16108 (1996).

[22] K. Harada, O. Kamimura, H. Kasai, T. Matsuda, A. Tonomura, and V. V. Moshchalkov, Science 274, 1167 (1996).

[23] A. Schmid and W. Hauger, J. Low Temp. Phys. 11, 667 (1973).

[24] U. Yaron, P. L. Gammel, D. A. Huse, R. N. Kleiman, C. S. Oglesby, E. Bucher, B. Batlogg, D. J. Bishop, K. Mortensen, K. Claussen, C. A. Bolle, and F. de la Cruz, Phys. Rev. Lett. 73, 2748 (1994). 